\title{
The Kirchhoff Index of Some Combinatorial Networks
}

\author{
Jia-Bao Liu, ${ }^{1,2,3}$ Xiang-Feng Pan, ${ }^{2}$ Jinde Cao, ${ }^{3,4}$ and Fu-Tao $\mathrm{Hu}^{2}$ \\ ${ }^{1}$ Department of Public Courses, Anhui Xinhua University, Hefei 230088, China \\ ${ }^{2}$ School of Mathematical Sciences, Anhui University, Hefei 230601, China \\ ${ }^{3}$ Department of Mathematics, Southeast University, Nanjing 210096, China \\ ${ }^{4}$ Department of Mathematics, Faculty of Science, King Abdulaziz University, Jeddah 21589, Saudi Arabia \\ Correspondence should be addressed to Jinde Cao; jdcao@seu.edu.cn
}

Received 7 December 2014; Revised 6 March 2015; Accepted 12 March 2015

Academic Editor: Carmen Coll

Copyright (C) 2015 Jia-Bao Liu et al. This is an open access article distributed under the Creative Commons Attribution License, which permits unrestricted use, distribution, and reproduction in any medium, provided the original work is properly cited.

\begin{abstract}
The Kirchhoff index $\operatorname{Kf}(G)$ is the sum of the effective resistance distances between all pairs of vertices in $G$. The hypercube $Q_{n}$ and the folded hypercube $F Q_{n}$ are well known networks due to their perfect properties. The graph $G^{*}$, constructed from $G$, is the line graph of the subdivision graph $S(G)$. In this paper, explicit formulae expressing the Kirchhoff index of $\left(Q_{n}\right)^{*}$ and $\left(F Q_{n}\right)^{*}$ are found by deducing the characteristic polynomial of the Laplacian matrix of $G^{*}$ in terms of that of $G$.
\end{abstract}

\section{Introduction}

It is well known that interconnection networks play an important role in parallel communication systems. An interconnection network is usually modelled by a connected graph $G=(V(G), E(G))$, where $V(G)$ denotes the set of processors and $E(G)$ denotes the set of communication links between processors in networks. The hypercube $Q_{n}$ and the folded hypercube $F Q_{n}$ are two very popular and efficient interconnection networks due to their excellent performance in some practical applications. The symmetry, regular structure, strong connectivity, small diameter, and many of their properties have been explored [1-5].

The adjacency matrix $A(G)$ of $G$ is an $n \times n$ matrix with the $(i, j)$-entry equal to 1 if vertices $i$ and $j$ are adjacent and to 0 if otherwise. Let $D(G)$ be the degree diagonal matrix of $G$, and $L(G)=D(G)-A(G)$ is called the Laplacian matrix of $G$. Denote the Laplacian characteristic polynomial of $G$ by $\sigma_{L(G)}(x)=\operatorname{det}(x I-L(G))=x^{n}+q_{1}(G) x^{n-1}+q_{2}(G) x^{n-2}+$ $\cdots+q_{n-1}(G) x+q_{n}(G)$, where $q_{i}(i=1,2, \ldots, n)$ are the coefficients of the Laplacian characteristic polynomial [6]. The eigenvalues of $A(G)$ and $L(G)$ are called eigenvalues and Laplacian eigenvalues of $G$, respectively. In this paper we are concerned with some finite undirected connected simple graphs (networks). For the underlying graph, theoretical definitions, and notations, we follow [7].

Let $G$ be a graph with vertices labelled $1,2, \ldots, n$. It is well known that the standard distance between two vertices of $G$, denoted by $d_{i j}$, is the shortest path connecting the two vertices. A novel distance function named resistance distance was firstly proposed by Klein and Randić [8]. The resistance distance between vertices $i$ and $j$, denoted by $r_{i j}$, is defined to be the effective electrical resistance between them if each edge of $G$ is replaced by a unit resistor [8]. A famous distance-based topological index as the Kirchhoff index, $\operatorname{Kf}(G)=(1 / 2) \sum_{i=1}^{n} \sum_{j=1}^{n} r_{i j}(G)$, is defined as the sum of resistance distances between all pairs of vertices in $G$ [8].

The Kirchhoff index has been attracting extensive attention due to its wide applications in physics, chemistry, graph theory, and so forth [9-18]. Details on its theory can be found in recent papers $[19,20]$ and the references cited therein. But there are only few works appearing on the Kirchhoff index in combinatorial networks. In the present paper, we establish the closed-form formulae expressing the Kirchhoff index of $\left(Q_{n}\right)^{*}$ and $\left(F Q_{n}\right)^{*}$, where the graph $G^{*}$, constructed from $G$, is the line graph of the subdivision graph $S(G)$.

The main purpose of this paper is to investigate the Kirchhoff index of some combinatorial networks. The graph 
$G^{*}$, constructed from $G$, is the line graph of the subdivision graph $S(G)$. We have established the relationships between $Q_{n}, F Q_{n}$ and their variant networks $\left(Q_{n}\right)^{*},\left(F Q_{n}\right)^{*}$, in terms of Kirchhoff index, respectively. Moreover, explicit formulae have been proposed for expressing the Kirchhoff index of $\left(Q_{n}\right)^{*}$ and $\left(F Q_{n}\right)^{*}$ by making use of the characteristic polynomial of the Laplacian matrix in spectral graph theory.

The remainder of the paper is organized as follows. Section 2 provides some underlying definitions and preliminaries in our discussion. The proofs of main results and some examples are given in Sections 3 and 4, respectively.

\section{Definitions and Preliminaries}

In this section, we recall some underlying definitions and properties which we need to use in the proofs of our main results as follows.

Definition 1 (hypercube $Q_{n}[21]$ ). The hypercube $Q_{n}$ has $2^{n}$ vertices each labelled with a binary string of length $n$. Two vertices $X=x_{1} x_{2} \cdots x_{n}$ and $Y=y_{1} y_{2} \cdots y_{n}$ are adjacent if and only if there exists an $i, 1 \leq i \leq n$, such that $x_{i}=\overline{y_{i}}$, where $\overline{y_{i}}$ denoted the complement of binary digit $y_{i}$ and $x_{j}=y_{j}$, for all $j \neq i$, and $1 \leq j \leq n$.

Definition 2 (folded hypercube $F Q_{n}[2]$ ). The folded hypercube $F Q_{n}$ can be constructed from $Q_{n}$ by adding an edge to every pair of vertices with complementary addresses. Two vertices $X=x_{1} x_{2} \cdots x_{n}$ and $\bar{X}=\overline{x_{1}} \overline{x_{2}} \cdots \overline{x_{n}}$ are adjacent in the folded hypercube $F Q_{n}$.

Definition 3 (construction of $G^{*}$ [22]). Define the following operation of $G$, constructing $G^{*}$ from $G$, as follows [22]:

(i) Replace each vertex $u \in V(G)$ by $K_{(u)}$, the complete graph on $\operatorname{deg}_{G}(u)$ vertices.

(ii) There is an edge joining a vertex of $K_{\left(u_{1}\right)}$ and a vertex of $K_{\left(u_{2}\right)}$ in $G^{*}$ if and only if there is an edge joining $u_{1}$ and $u_{2}$ in $G$.

(iii) For each vertex $v$ of $K_{(u)}, \operatorname{deg}_{G^{*}}(v)=\operatorname{deg}_{G}(u)$.
Recall the following two underlying conceptions that related to the above construction of $G^{*}$. The subdivision graph $S(G)$ of a graph $G$ is obtained from $G$ by deleting every edge $u v$ of $G$ and replacing it by a vertex $w$ of degree 2 that is joined to $u$ and $v$ (see page 151 of [23]). The line graph of a graph $G$, denoted by $L(G)$, is the graph whose vertices correspond to the edges of $G$ with two vertices of $L(G)$ being adjacent if and only if the corresponding edges in $G$ share a common vertex [22].

It is amazing and interesting that $G^{*}$, constructed from $G$ as the graph operation above, is equivalent to the line graph of the subdivision graph $S(G)[22]$; that is, $G^{*} \cong L(S(G))$.

Remark 4. Note that there is an elementary and important property: if $G$ is an $r$-regular graph (combinatorial network), then $G^{*}$ is also an $r$-regular graph (combinatorial network); however, the topological structure of $G^{*}$ is quite more complicated than $G$; consequently, dealing with the problems of calculating Kirchhoff index of $\left(Q_{n}\right)^{*}$ and $\left(F Q_{n}\right)^{*}$ is not easy, even though we have handled the formulas for calculating the Kirchhoff index of $\left(Q_{n}\right)$ and $\left(F Q_{n}\right)$ in $[24,25]$.

Yin and Wang [26] have proved the following Lemma.

Lemma 5 (see [26]). For $Q_{n}$ with any integer $n \geq 2$, the spectrum of Laplacian matrix of $Q_{n}$ is

$$
\operatorname{Spec}_{L}\left(Q_{n}\right)=\left(\begin{array}{cccccc}
0 & 2 & \cdots & 2 i & \cdots & 2 n \\
C_{n}^{0} & C_{n}^{1} & \cdots & C_{n}^{i} & \cdots & C_{n}^{n}
\end{array}\right)
$$

where $2 i, i=0,1, \ldots, n$, are the eigenvalues of the Laplacian matrix of $Q_{n}$ and $C_{n}^{i}$ are the multiplicities of the eigenvalues $2 i$.

M. Chen and B. X. Chen have studied the Laplacian spectra of $F Q_{n}$ in [3].

Lemma 6 (see [3]). For $F Q_{n}$ with any integer $n \geq 2$, the spectra of Laplacian matrix of $F Q_{n}$ are as follows:

(1) If $n \equiv 0(\bmod 2)$, then

$$
\operatorname{Spec}_{L}\left(F Q_{n}\right)=\left(\begin{array}{ccccccc}
0 & 4 & 8 & \cdots & 2 n-8 & 2 n-4 & 2 n \\
C_{n}^{n} & C_{n}^{n-1}+C_{n}^{n-2} & C_{n}^{n-3}+C_{n}^{n-4} & \cdots & C_{n}^{5}+C_{n}^{4} & C_{n}^{3}+C_{n}^{2} & C_{n}^{1}+C_{n}^{0}
\end{array}\right)
$$

(2) If $n \equiv 1(\bmod 2)$, then

$\operatorname{Spec}_{L}\left(F Q_{n}\right)$

$$
=\left(\begin{array}{ccccccc}
0 & 4 & 8 & \cdots & 2 n-6 & 2 n-2 & 2 n+2 \\
C_{n}^{0} & C_{n}^{1}+C_{n}^{2} & C_{n}^{3}+C_{n}^{4} & \cdots & C_{n}^{n-4}+C_{n}^{n-3} & C_{n}^{n-2}+C_{n}^{n-1} & C_{n}^{n}
\end{array}\right),
$$

where $C_{n}^{i}$ are the binomial coefficients and the elements in the first and second rows are the Laplacian eigenvalues of $F Q_{n}$ and the multiplicities of the corresponding eigenvalues, respectively.
Lemma 7 (see $[11,27])$. Let $G$ be a connected graph, with $n \geq$ 2 vertices, and $\mu_{1} \geq \mu_{2} \geq \cdots \geq \mu_{n}=0$ are the Laplacian eigenvalues of $G$; then

$$
K f(G)=n \sum_{i=1}^{n-1} \frac{1}{\lambda_{i}}
$$

Let $\sigma_{(G)}(x)$ be the characteristic polynomial of the Laplacian matrix of a graph $G$; the following results were shown in [28]. 
Lemma 8 (see [28]). Let $G$ be an r-regular connected graph with $n$ vertices and $m$ edges; then

$$
\begin{aligned}
& \sigma_{L(G)}(x)=(x-2 r)^{m-n} \sigma_{G}(x), \\
& \sigma_{S(G)}(x)=(-1)^{m}(2-x)^{m-n} \sigma_{G}(x(r+2-x)),
\end{aligned}
$$

where $\sigma_{L(G)}(x)$ and $\sigma_{S(G)}(x)$ are the characteristic polynomials for the Laplacian matrix of graphs $L(G)$ and $S(G)$, respectively.

Let $G$ be a bipartite graph with a bipartition; $V(G)=$ $(U, V)$ is called an $(r, s)$-semiregular graph if all vertices in $U$ have degree $r$ and all vertices in $V$ have degree $s$.

Lemma 9 (see [29]). Let $G$ be an $(r, s)$-semiregular connected graph with $n$ vertices and $m$ edges, and $\sigma_{L(G)}(x)$ is the Laplacian characteristic polynomial of the line graph $L(G)$. Then

$$
\sigma_{L(G)}(x)=(-1)^{n}(x-(r+s))^{m-n} \sigma_{G}(r+s-x) .
$$

\section{Main Results}

Theorem 10. For $\left(Q_{n}\right)^{*}$ with any integer $n \geq 2$, one has

$$
\begin{aligned}
K f\left(\left(Q_{n}\right)^{*}\right)= & \left(n^{2}+2 n\right) K f\left(Q_{n}\right) \\
& +\frac{\left(n^{2}-n-2\right) 4^{n}+2^{n} n}{n+2} .
\end{aligned}
$$

Proof. Notice that $Q_{n}$ is $n$-regular graph with $2^{n}$ vertices and $n 2^{n-1}$ edges. Suppose that $S\left(Q_{n}\right)$ has $u$ vertices and $w$ edges, for convenience, and denote the degree of vertices in $Q_{n}$ by $d$. Obviously, $u=2^{n}+2^{n-1} d$ and $w=2^{n} d$, respectively.

From Lemma 9, we can get

$$
\begin{aligned}
& \sigma_{L\left(S\left(\mathrm{Q}_{n}\right)\right)}(x) \\
& \quad=(-1)^{u}(x-(d+2))^{w-u} \sigma_{S\left(\mathrm{Q}_{n}\right)}(d+2-x) .
\end{aligned}
$$

By virtue of Lemma 8 , it follows that

$$
\begin{aligned}
& \sigma_{S\left(Q_{n}\right)}(x) \\
& \quad=(-1)^{2^{n-1} d}(2-x)^{2^{n-1} d-2^{n}} \sigma_{Q_{n}}(x(d+2-x)) .
\end{aligned}
$$

Replacing $x$ with $d+2-x$ in (9), we have

$$
\begin{aligned}
& \sigma_{S\left(\mathrm{Q}_{n}\right)}(d+2-x) \\
& \quad=(-1)^{2^{n-1} d}(x-d)^{2^{n-1} d-2^{n}} \sigma_{\mathrm{Q}_{n}}(x(d+2-x)) .
\end{aligned}
$$

Substituting (8) with (10), the Laplacian characteristic polynomial of $L\left(S\left(Q_{n}\right)\right)$ is

$$
\begin{aligned}
\sigma_{L\left(S\left(\mathrm{Q}_{n}\right)\right)}(x)= & (-1)^{u+2^{n-1} d}(x-d)^{2^{n-1} d-2^{n}} \\
& \cdot(x-(d+2))^{w-u} \sigma_{\mathrm{Q}_{n}}(x(d+2-x)) .
\end{aligned}
$$

From the definition graph $\left(Q_{n}\right)^{*}$ and (11), one can immediately obtain

$$
\begin{aligned}
\sigma_{\left(\mathrm{Q}_{n}\right)^{*}}(x)= & (-1)^{u+2^{n-1} d}(x-d)^{2^{n-1} d-2^{n}}(x-(d+2))^{w-u} \\
& \cdot \sigma_{\mathrm{Q}_{n}}(x(d+2-x)) .
\end{aligned}
$$

Combining (12), $u=2^{n}+2^{n-1} d, w=2^{n} d$, and $d=n$, it holds that

$$
\begin{aligned}
& \sigma_{\left(Q_{n}\right)^{*}}(x)=(-1)^{2^{n}(d+1)}(x-d)^{2^{n-1}(n-2)} \\
& \cdot(x-(d+2))^{2^{n-1}(n-2)} \sigma_{Q_{n}}(x(d+2-x)) \\
& =(-1)^{2^{n}(n+1)}(x-n)^{2^{n-1}(n-2)}(x-(n+2))^{2^{n-1}(n-2)} \\
& \cdot \sigma_{Q_{n}}(x(n+2-x)) .
\end{aligned}
$$

Since the roots of $x(n+2-x)=\mu_{i}$ are

$$
\begin{aligned}
& x_{1, i}=\frac{n+2-\sqrt{(n+2)^{2}-4 \mu_{i}}}{2}, \\
& x_{2, i}=\frac{n+2+\sqrt{(n+2)^{2}-4 \mu_{i}}}{2},
\end{aligned}
$$

where $\mu_{1} \geq \mu_{2} \cdots \geq \mu_{2^{n}}=0$ are the Laplacian eigenvalues of $Q_{n}$.

It follows from (12) that the Laplacian spectrum of $\left(Q_{n}\right)^{*}$ is

$$
\begin{aligned}
& \operatorname{Spec}_{L}\left(\left(Q_{n}\right)^{*}\right) \\
& =\left\{\frac{n, n, \ldots, n}{2^{n-1}(n-2)}, \frac{n+2, n+2, \ldots, n+2}{2^{n-1}(n-2)}\right\} \\
& \cdot \bigcup_{i=1}^{2^{n}}\left\{\frac{n+2-\sqrt{(n+2)^{2}-4 \mu_{i}}}{2}\right\} \\
& \cdot \bigcup_{i=1}^{2^{n}}\left\{\frac{n+2+\sqrt{(n+2)^{2}-4 \mu_{i}}}{2}\right\} \text {. }
\end{aligned}
$$

Noticing that $\left(Q_{n}\right)^{*}$ has $2^{n} n$ vertices, we get the following result from Lemmas 5 and 7 and (15). Therefore,

$$
\begin{gathered}
\mathrm{Kf}\left(\left(Q_{n}\right)^{*}\right)=2^{n} n\left(\frac{2^{n-1}(n-2)}{n}+\frac{2^{n-1}(n-2)}{n+2}\right. \\
\left.+\sum_{i=1}^{2^{n}-1} \frac{1}{x_{1, i}}+\sum_{i=1}^{2^{n}} \frac{1}{x_{2, i}}\right)=\frac{4^{n}\left(n^{2}-n-2\right)}{n+2} \\
+2^{n} n\left(\sum_{i=1}^{2^{n}-1} \frac{2}{n+2-\sqrt{(n+2)^{2}-4 \mu_{i}}}\right.
\end{gathered}
$$




$$
\begin{aligned}
& \left.+\sum_{i=1}^{2^{n}} \frac{2}{n+2+\sqrt{(n+2)^{2}-4 \mu_{i}}}\right)=\frac{4^{n}\left(n^{2}-n-2\right)}{n+2} \\
& +2^{n} n\left(\sum_{i=1}^{2^{n}-1} \frac{2}{n+2-\sqrt{(n+2)^{2}-4 \mu_{i}}}\right. \\
& \left.+\sum_{i=1}^{2^{n}-1} \frac{2}{n+2+\sqrt{(n+2)^{2}-4 \mu_{i}}}+\frac{1}{n+2}\right) \\
& =\frac{4^{n}\left(n^{2}-n-2\right)}{n+2}+\frac{2^{n} n}{n+2}+2^{n} n \sum_{i=1}^{2^{n}-1} \frac{n+2}{\mu_{i}} \\
& =\frac{\left(n^{2}-n-2\right) 4^{n}+2^{n} n}{n+2}+n(n+2) 2^{2^{n}} \sum_{i=1}^{n} \frac{1}{\mu_{i}}=\left(n^{2}\right. \\
& +2 n) \operatorname{Kf}\left(Q_{n}\right)+\frac{\left(n^{2}-n-2\right) 4^{n}+2^{n} n}{n+2} .
\end{aligned}
$$

This completes the proof.

The following theorem [24] provided the closed-form formula expressing the Kirchhoff index of $Q_{n}$ with any integer $n \geq 2$.
Theorem 11 (see [24]). Let $C_{n}^{i}$ be the binomial coefficients for $Q_{n}$ with any integer $n \geq 2$. Then

$$
K f\left(Q_{n}\right)=2^{n} \sum_{i=1}^{n} \frac{C_{n}^{i}}{2 i} .
$$

Theorem 12. Let $C_{n}^{i}$ be the binomial coefficients for $\left(Q_{n}\right)^{*}$ with any integer $n \geq 2$. Then

$$
\begin{aligned}
K f\left(\left(Q_{n}\right)^{*}\right)= & \left(n^{2}+2 n\right) 2^{n} \sum_{i=1}^{n} \frac{C_{n}^{i}}{2 i} \\
& +\frac{\left(n^{2}-n-2\right) 4^{n}+n 2^{n}}{n+2} .
\end{aligned}
$$

Proof. From Theorems 10 and 11 one can immediately arrive at the explicit formula expressing the Kirchhoff index of $\left(Q_{n}\right)^{*}$ with any integer $n$.

Remark 13. Theorem 11 gives the value of $\operatorname{Kf}\left(Q_{n}\right)$ in a nice closed-form formula. In [30] a similar, slightly more involved, closed-form formula was given, and, moreover, an asymptotic value of $2^{2 d} / d$ was given for $\operatorname{Kf}\left(Q_{n}\right)$. Comparing the asymptotic relative sizes of $\operatorname{Kf}\left(Q_{n}\right)$ and $\operatorname{Kf}\left(\left(Q_{n}\right)^{*}\right)$ in the present article, the latter is much larger than the former.

In the following, we will further address the Kirchhoff index of $\left(F Q_{n}\right)^{*}$. Primarily, notice that $F Q_{n}$ is a regular graph with degree for any vertex and the Laplacian spectrum of $F Q_{n}$ is as follows:

(1) If $n \equiv 0(\bmod 2)$, then

$$
\operatorname{Spec}_{L}\left(F Q_{n}\right)=\left(\begin{array}{ccccccc}
0 & 4 & 8 & \cdots & 2 n-8 & 2 n-4 & 2 n \\
C_{n}^{n} & C_{n}^{n-1}+C_{n}^{n-2} & C_{n}^{n-3}+C_{n}^{n-4} & \cdots & C_{n}^{5}+C_{n}^{4} & C_{n}^{3}+C_{n}^{2} & C_{n}^{1}+C_{n}^{0}
\end{array}\right) .
$$

(2) If $n \equiv 1(\bmod 2)$, then

$$
\operatorname{Spec}_{L}\left(F Q_{n}\right)=\left(\begin{array}{ccccccc}
0 & 4 & 8 & \cdots & 2 n-6 & 2 n-2 & 2 n+2 \\
C_{n}^{0} & C_{n}^{1}+C_{n}^{2} & C_{n}^{3}+C_{n}^{4} & \cdots & C_{n}^{n-4}+C_{n}^{n-3} & C_{n}^{n-2}+C_{n}^{n-1} & C_{n}^{n}
\end{array}\right) .
$$

In an almost identical way as Theorem 10, we derive the following formula expressing the Kirchhoff index of $\left(F Q_{n}\right)^{*}$. The proof is omitted here for the completely similar deduction to Theorem 10.

Theorem 14. For $\left(F Q_{n}\right)^{*}$ with any integer $n \geq 2$, one has

$$
\begin{aligned}
K f\left(\left(F Q_{n}\right)^{*}\right)= & \left(n^{2}+4 n+3\right) K f\left(F Q_{n}\right) \\
& +\frac{\left(n^{2}+n-2\right) 4^{n}+(n+1) 2^{n}}{n+3} .
\end{aligned}
$$

In [25], the authors have proposed the following Kirchhoff index of $F Q_{n}$ with any integer $n \geq 2$.

Theorem 15 (see [25]). Let $C_{n}^{i}$ denote the binomial coefficients for $F Q_{n}$ with any integer $n \geq 2$. Then

(1) $K f\left(F Q_{n}\right)=2^{n} \sum_{i=1}^{n / 2}\left(\left(C_{n}^{n-i}+C_{n}^{n-i-1}\right) / 4 i\right), i=$ $1,2, \ldots, n / 2$, if $n \equiv 0(\bmod 2)$,

(2) $K f\left(F Q_{n}\right)=2^{n} \sum_{i=1}^{(n-1) / 2}\left(\left(C_{n}^{2 i-1}+C_{n}^{2 i}\right) / 4 i\right)+2^{n-1} /(n+1)$, $i=1,2, \ldots,(n-1) / 2$, if $n \equiv 1(\bmod 2)$. 
Theorem 16. Let $C_{n}^{i}$ denote the binomial coefficients for $\left(F Q_{n}\right)^{*}$ with any integer $n \geq 2$. Then

(1) $K f\left(\left(F Q_{n}\right)^{*}\right)=\left(n^{2}+4 n+3\right) 2^{n} \sum_{i=1}^{n / 2}\left(\left(C_{n}^{n-i}+C_{n}^{n-i-1}\right) / 4 i\right)+$ $\left(\left(n^{2}+n-2\right) 4^{n}+(n+1) 2^{n}\right) /(n+3), i=1,2, \ldots, n / 2$, if $n \equiv 0(\bmod 2)$,

(2) $K f\left(\left(F Q_{n}\right)^{*}\right)=\left(n^{2}+4 n+3\right) 2^{n} \sum_{i=1}^{(n-1) / 2}\left(\left(C_{n}^{2 i-1}+\right.\right.$ $\left.\left.C_{n}^{2 i}\right) / 4 i\right)+\left(\left(n^{2}+n-2\right) 4^{n}+(n+1) 2^{n}\right) /(n+3)+(n+3) 2^{n-1}$, $i=1,2, \ldots,(n-1) / 2$, if $n \equiv 1(\bmod 2)$.

Proof. From Theorems 14 and 15, it is not difficult to deduce the above formula expressing the Kirchhoff index of $\left(Q_{n}\right)^{*}$ with any integer $n$.

Remark 17. Theorems 12 and 16 have presented a method to calculate the Kirchhoff index of $\left(Q_{n}\right)^{*}$ and $\left(F Q_{n}\right)^{*}$, which is difficult to calculate directly. We found a relationship between the graph $G$ and $G^{*}$ by deducing the characteristic polynomial of the Laplacian matrix and obtained the Laplacian spectrum of $\operatorname{Kf}\left(\left(Q_{n}\right)^{*}\right)$ and $\operatorname{Kf}\left(\left(F Q_{n}\right)^{*}\right)$. If we can compute the Kirchhoff index $\operatorname{Kf}(G)$ readily, then, by Laplacian spectrum of $G^{*}$, we can also obtain the Kirchhoff index $\operatorname{Kf}\left(G^{*}\right)$ which is hard to calculate immediately. Furthermore, utilizing this approach one can also formulate the Kirchhoff index of other general graphs.

\section{Some Examples}

To demonstrate the theoretical analysis, we provide some examples in this subsection, which are an application of our results. Without loss of generality, we suppose that the case is $n=2$ for simplicity. Obviously, $\left|V\left(Q_{2}\right)\right|=4$, and the eigenvalues of the Laplacian matrix of $Q_{2}$ are $\lambda_{1}=4, \lambda_{2}=$ $\lambda_{3}=2$, and $\lambda_{4}=0$. Based on Lemma 7 , it is easy to obtain that

$$
\mathrm{Kf}\left(Q_{2}\right)=4 \cdot \sum_{i=1}^{3} \frac{1}{\lambda_{i}}=4 \cdot\left(\frac{1}{\lambda_{1}}+\frac{1}{\lambda_{2}}+\frac{1}{\lambda_{3}}\right)=5 .
$$

According to the consequence of Theorem 10, one can readily derive that

$$
\operatorname{Kf}\left(\left(Q_{2}\right)^{*}\right)=8 \mathrm{Kf}\left(Q_{2}\right)+2=42
$$

On the other hand, we use another approach to calculate $\operatorname{Kf}\left(\left(Q_{2}\right)^{*}\right)$. For a circulant graph $G$, the authors of [31] showed that

$$
n-1 \leq \operatorname{Kf}(G) \leq \frac{n^{3}-n}{12} .
$$

The first equality holds if and only if $G$ is $K_{n}$ and the second does if and only if $G$ is $C_{n}$.

By virtue of the definition of $G^{*}$, it is not difficult to get that $\left(Q_{2}\right)^{*} \cong C_{8}$.

Consequently, the same Kirchhooff index can be drawn as follows:

$$
\mathrm{Kf}\left(\left(Q_{2}\right)^{*}\right)=\frac{n^{3}-n}{12}=\frac{8^{3}-8}{12}=42
$$

As the application of Theorem 14, we proceed to derive that $\mathrm{Kf}\left(\left(F Q_{2}\right)^{*}\right)$.

Note that the eigenvalues of the Laplacian matrix of $F Q_{2}$ are $\lambda_{1}=\lambda_{2}=\lambda_{3}=4$, and $\lambda_{4}=0$. Based on Lemma 7, we have

$$
\mathrm{Kf}\left(F Q_{2}\right)=4 \cdot \sum_{i=1}^{3} \frac{1}{\lambda_{i}}=4 \cdot\left(\frac{1}{\lambda_{1}}+\frac{1}{\lambda_{2}}+\frac{1}{\lambda_{3}}\right)=3
$$

Similarly, according to the consequence of Theorem 14, it holds that

$$
\mathrm{Kf}\left(\left(F Q_{2}\right)^{*}\right)=15 \mathrm{Kf}\left(F Q_{n}\right)+\frac{76}{5}=60.2
$$

Summing up the examples, the results above coincide the fact, which show our theorems are correct and effective.

\section{Conflict of Interests}

The authors declare that there is no conflict of interests regarding the publication of this paper.

\section{Acknowledgments}

The work of J. B. Liu was supported by Anhui Provincial Natural Science Foundation under Grant no. KJ2013B105 and the National Science Foundation of China under Grant nos. 11471016 and 11401004. The work of F. T. Hu was supported by Anhui Provincial Natural Science Foundation (1408085QA03) and the National Science Foundation of China under Grant no. 11401004. The authors would like to express their sincere gratitude to the anonymous referees for their valuable suggestions, which led to a significant improvement of the original paper.

\section{References}

[1] M. Xu, X. D. Hu, and J. M. Xu, "Edge-pancyclicity and Hamiltonian laceability of the balanced hypercubes," Applied Mathematics and Computation, vol. 189, no. 2, pp. 1393-1401, 2007.

[2] A. El-Amawy and S. Latifi, "Properties and performance of folded hypercubes," IEEE Transactions on Parallel and Distributed Systems, vol. 2, no. 1, pp. 31-42, 1991.

[3] M. Chen and B. X. Chen, "Spectra of folded hypercubes," Journal of East China Normal University, vol. 2, no. 39, pp. 39-46, 2011.

[4] S. Cao, H. Liu, and X. He, "On constraint fault-free cycles in folded hypercube," International Journal of Applied Mathematics and Statistics, vol. 42, no. 12, pp. 38-44, 2013.

[5] J.-B. Liu, X.-F. Pan, and J. Cao, "Some properties on Estrada index of folded hypercubes networks," Abstract and Applied Analysis, vol. 2014, Article ID 167623, 6 pages, 2014.

[6] W. Qiu and W. Yan, “The coefficients of Laplacian characteristic polynomials of graphs," Linear Algebra and Its Applications, vol. 436, no. 7, pp. 2474-2479, 2012.

[7] J. M. Xu, Topological Strucure and Analysis of Interconnction Networks, Kluwer Academic Publishers, Dordrecht, The Netherlands, 2001. 
[8] D. J. Klein and M. Randić, "Resistance distance," Journal of Mathematical Chemistry, vol. 12, no. 1-4, pp. 81-95, 1993.

[9] L. Ye, "On the Kirchhoff index of some toroidal lattices," Linear and Multilinear Algebra, vol. 59, no. 6, pp. 645-650, 2011.

[10] B. Zhou and N. Trinajstić, "A note on Kirchhoff index," Chemical Physics Letters, vol. 455, no. 1-3, pp. 120-123, 2008.

[11] H.-Y. Zhu, D. J. Klein, and I. Lukovits, "Extensions of the Wiener number," Journal of Chemical Information and Computer Sciences, vol. 36, no. 3, pp. 420-428, 1996.

[12] Z. You, L. You, and W. Hong, "Comment on Kirchhoff index in line, subdivision and total graphs of a regular graph," Discrete Applied Mathematics, vol. 161, no. 18, pp. 3100-3103, 2013.

[13] W. J. Xiao and I. Gutman, "Resistance distance and Laplacian spectrum," Theoretical Chemistry Accounts, vol. 110, no. 4, pp. 284-289, 2003.

[14] E. Estrada and N. Hatano, "Topological atomic displacements, Kirchhoff and Wiener indices of molecules," Chemical Physics Letters, vol. 486, no. 4-6, pp. 166-170, 2010.

[15] A. D. Maden, A. S. Cevik, I. N. Cangul, and K. C. Das, "On the KIRchhoff matrix, a new KIRchhoff index and the KIRchhoff energy," Journal of Inequalities and Applications, vol. 2013, article 337, pp. 83-94, 2013.

[16] J. B. Liu, X. F. Pan, J. Cao, and F. F. Hu, "A note on 'some physical and chemical indices of clique-inserted lattices,' Journal of Statistical Mechanics: Theory and Experiment, vol. 10, no. 6, pp. $1-8,2014$.

[17] J.-B. Liu, X.-F. Pan, F.-T. Hu, and F.-F. Hu, "Asymptotic Laplacian-energy-like invariant of lattices," Applied Mathematics and Computation, vol. 253, pp. 205-214, 2015.

[18] J.-B. Liu and X.-F. Pan, "Asymptotic incidence energy of lattices," Physica A. Statistical Mechanics and its Applications, vol. 422, pp. 193-202, 2015.

[19] C. Arauz, "The Kirchhoff indexes of some composite networks," Discrete Applied Mathematics, vol. 160, no. 10-11, pp. 1429-1440, 2012.

[20] M. Bianchi, A. Cornaro, J. L. Palacios, and A. Torriero, "Bounds for the Kirchhoff index via majorization techniques," Journal of Mathematical Chemistry, vol. 51, no. 2, pp. 569-587, 2013.

[21] R. Indhumathi and S. A. Choudum, "Embedding certain height-balanced trees and complete $\mathrm{p}^{m}$-ary trees into hypercubes," Journal of Discrete Algorithms, vol. 22, pp. 53-65, 2013.

[22] D. J. Klein and E. Yi, "A comparison on metric dimension of graphs, line graphs, and line graphs of the subdivision graphs," European Journal of Pure and Applied Mathematics, vol. 5, no. 3, pp. 302-316, 2012.

[23] G. Chartrand and P. Zhang, Introduction to Graph Theory, McGraw-Hill, Kalamazoo, Mich, USA, 2004.

[24] J. Liu, J. Cao, X.-F. Pan, and A. Elaiw, "The Kirchhoff index of hypercubes and related complex networks," Discrete Dynamics in Nature and Society, vol. 2013, Article ID 543189, 7 pages, 2013.

[25] J. Liu, X.-F. Pan, Y. Wang, and J. Cao, "The Kirchhoff index of folded hypercubes and some variant networks," Mathematical Problems in Engineering, vol. 2014, Article ID 380874, 9 pages, 2014.

[26] J. H. Yin and R. G. Wang, "Spectra of Laplacian matrices of hypercubes," Journal of Zhejiang University. Science Edition, vol. 34, no. 3, pp. 321-323, 2007.

[27] I. Gutman and B. Mohar, "The quasi-Wiener and the Kirchhoff indices coincide," Journal of Chemical Information and Computer Sciences, vol. 36, no. 5, pp. 982-985, 1996.
[28] X. Gao, Y. Luo, and W. Liu, "Kirchhoff index in line, subdivision and total graphs of a regular graph," Discrete Applied Mathematics, vol. 160, no. 4-5, pp. 560-565, 2012.

[29] B. Mohar and Y. Alavi, "The Laplacian spectrum of graphs," in Graph Theory, Combinatorics, and Applications, vol. 2, pp. 871898, Wiley-Interscience, 1991.

[30] J. L. Palacios and J. M. Renom, "Bounds for the Kirchhoff index of regular graphs via the spectra of their random walks," International Journal of Quantum Chemistry, vol. 110, no. 9, pp. 1637-1641, 2010.

[31] H. P. Zhang and Y. J. Yang, "Resistance distance and kirchhoff index in circulant graphs," International Journal of Quantum Chemistry, vol. 107, no. 2, pp. 330-339, 2007. 


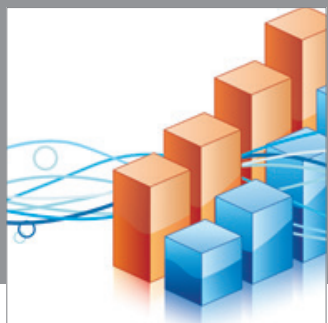

Advances in

Operations Research

mansans

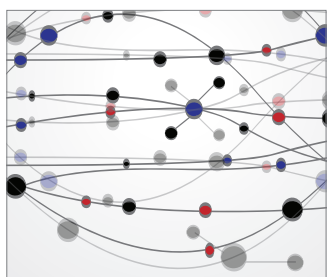

The Scientific World Journal
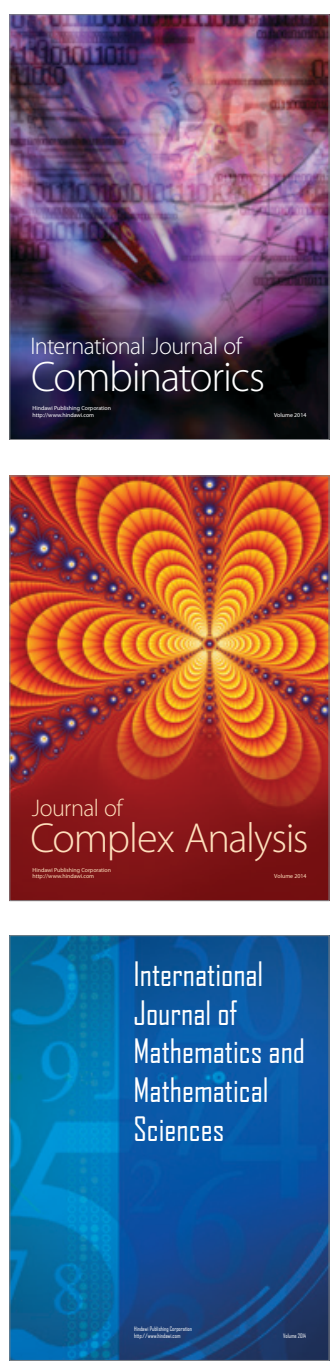
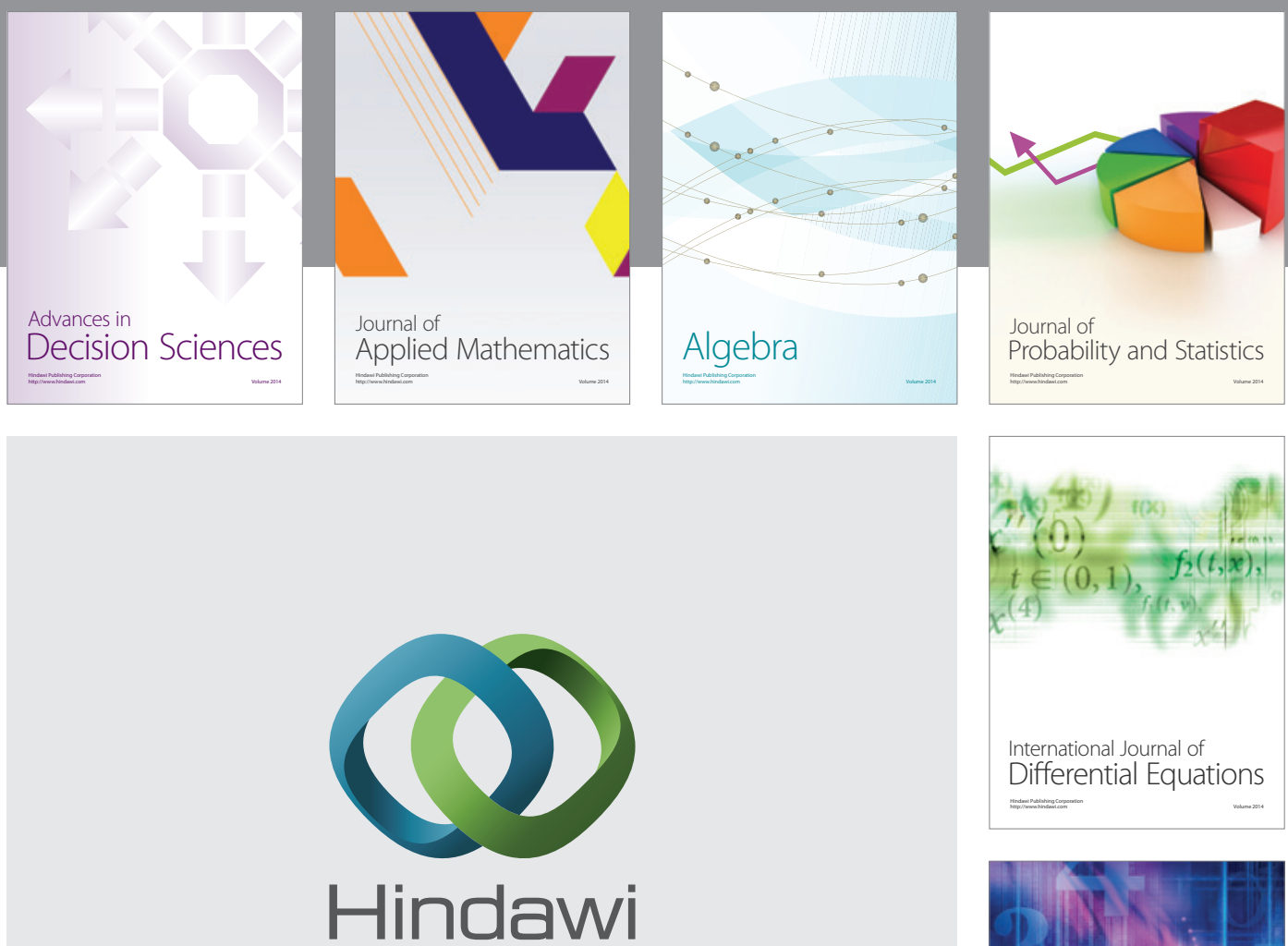

Submit your manuscripts at http://www.hindawi.com
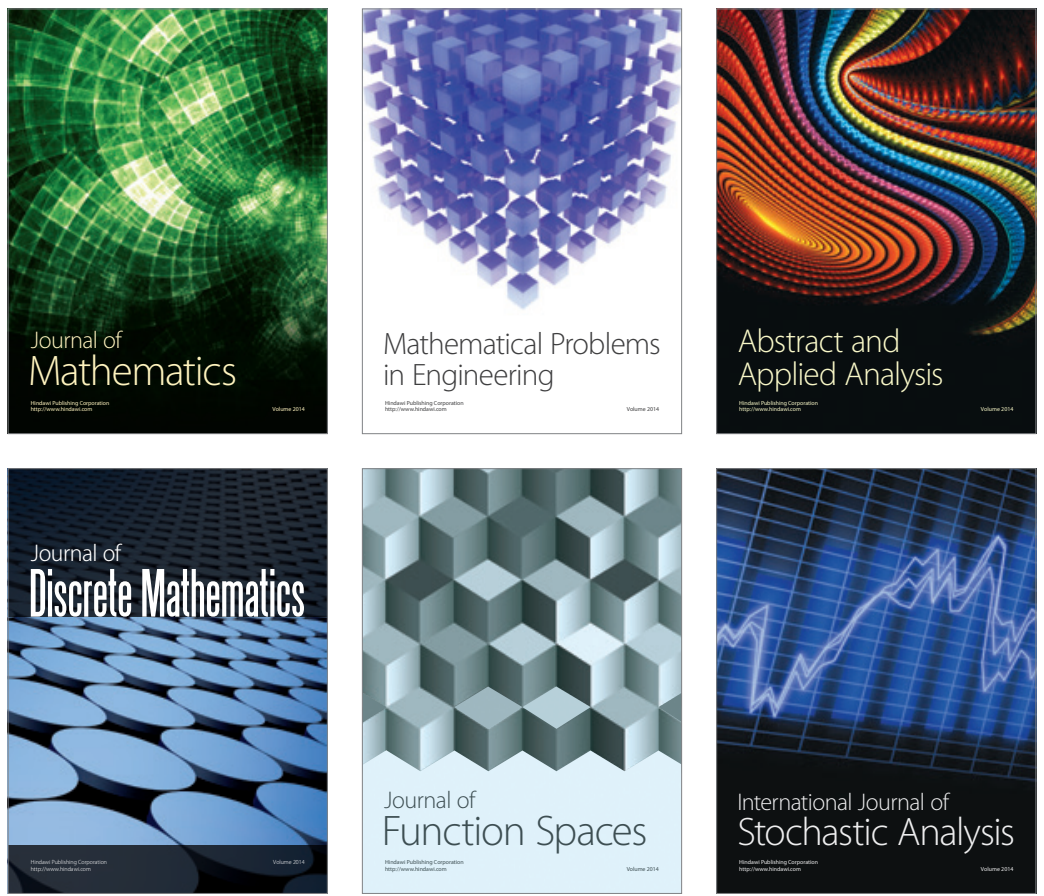

Journal of

Function Spaces

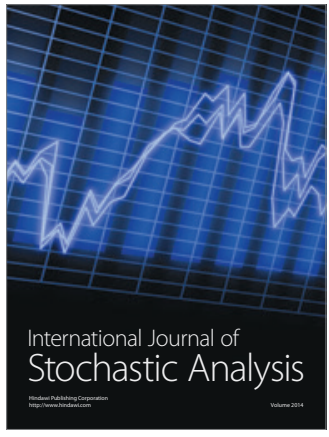

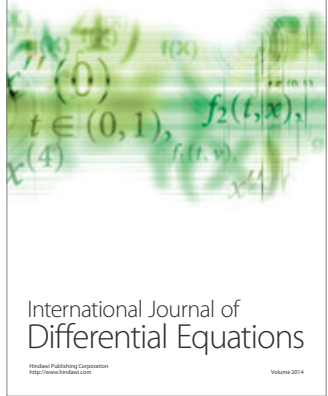
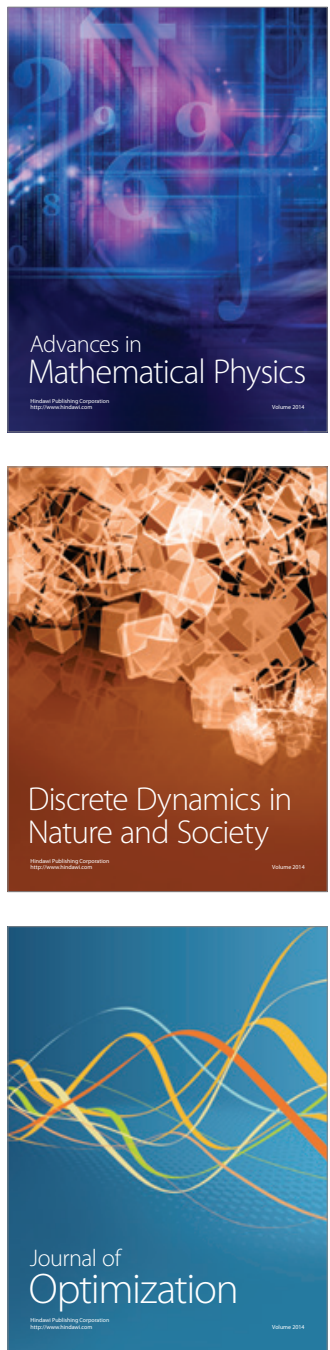